\title{
Conscious beyond ourselves
}

Stuart Sutherland

Animal Thinking.

By Donald R. Griffin.

Harvard University Press: 1984. Pp. 237.

$\$ 19.25$, f15.50.

THE ascription of consciousness to other beings surely depends on their similarity to ourselves, and in particular on how far their activities resemble those of our own that are consciously conducted. The relevant similarities are, however, not merely those of behaviour. Most people would be reluctant to think of a robot as conscious no matter how purposeful its activities, so long as its body was made from metal plates and its brain and nervous system from electronic components. Indeed, when it comes to consciousness, it is doubtful whether there can be any substitute for flesh and blood. For these reasons, most people would have no hesitation in thinking of other primates as conscious and would probably extend the attribute to many other mammals. There is no clear point in the animal kingdom where it is possible to be sure that consciousness stops, though paramecia and even ants are so different from ourselves that if they have some form of consciousness one feels it is likely to be very different from our own both in degree and in kind. It is of course equally difficult to decide at what point the growing fetus develops consciousness: to judge from a baby's undifferentiated behaviour, it may be many months after birth before it achieves the sort of awareness an adult has.

In Animal Thinking, a coda to his previous book The Question of Animal A wareness (second edition, 1981), Donald Griffin suggests that virtually all animals with a nervous system are conscious. He does not define consciousness since, as he rightly observes, such definitions are circular involving the use of terms like intention, idea, notion, awareness and so on. In trying to decide how far down the animal kingdom consciousness extends, he appears to use three criteria.

The first and most compelling is that an animal should exhibit "versatile adaptability of behaviour to changing circumstances and challenges". The most enjoyable and instructive parts of the book are those in which Griffin demonstrates the existence of this kind of behaviour by describing such activities as plovers drawing a predator away from their nests, ant-lions digging pits for prey and throwing sand at them, the construction of "house" cases by the larvae of caddis flies, and the recently learned ability of dolphins to escape from the nets used in tuna fishing. To the argument that such activities as the dance of the honey bee are largely genetic- ally determined, he replies rather weakly that even so they may involve an element of consciousness.

The word "may" occurs repeatedly throughout the book, particularly in connection with Griffin's second argument, which is that whenever an animal performs a complex activity, it might be useful for it to be conscious of what it is doing. In fact, this is less an argument than an assertion. As far as we know, there is no behaviour, no matter how complex, that could not be exhibited by an organism or by a computer without consciousness, and many of the unconscious calculations made in perception or in controlling motor activity appear to be as complex as the thinking of an Einstein.

Griffin draws his third argument from Nicholas Humphrey, who has suggested that to deal with their co-specifics social animals must empathize with one another. But again, empathy is simply using a model of oneself appropriately modified to fit someone else's circumstances in order to predict his goals, needs and behaviour.

\section{Resolution to do better}

\section{Akiyoshi Wada}

Methods of Protein and Nucleic Acid

Research, Vols 1 and 2.

By Lev A. Osterman.

Springer-Verlag: 1984,Vol.1 pp.342,

DM156, \$59. Vol.2 pp.204, DM98, \$38.

Ir IS difficult to over-estimate the contribution made by spectroscopic methods to the advancement of modern physics and chemistry during the past one hundred years. Electrophoresis and ultracentrifugation, the topics covered in Lev. A. Osterman's two-volume work, are two of the most important methods of molecular separation and analysis, and are today playing analogous roles in the development of molecular biology and biophysics.

Just as the essence of spectroscopy is the separation of photons according to their energies, the essence of the molecular techniques is to distinguish between molecules according to their physical and chemical characteristics. The results obtained from the two sets of methods are also similar one a set of bands on a photographic plate, representing molecular species, the other a series of peaks on recording paper showing the intensity of electromagnetic waves. In both cases the resolving power is a crucial factor in determining the quality of the result.

A further aspect of methods of molecular separation is their multidimensional nature. Any one of a variety of parameters influencing the migration velocity of molecules in the separation medium may be used - size, amount and sign of the electric
There is no obvious reason why such a model should be of use only if it appears in conscious awareness.

Griffin repeatedly urges that further research should be undertaken on animal consciousness, but he gives no indication of how to go about it. He also believes that only by understanding the consciousness of animals will it be possible to explain their behaviour. He forgets, however, that explanations in terms of consciousness are not rigorous since they rely on our manipulating a model of ourselves. To offer rigorous explanations it is necessary to specify the processes that underlie both a given sequence of thoughts and the organism's behaviour. It is worth asking whether the knowledge that a given species was conscious would really make any difference to how its behaviour is to be explained. The question is more difficult to answer than Griffin allows.

Stuart Sutherland is Director of the Centre for Research on Perception and Cognition at the University of Sussex. charge, buoyant density, affinity to a specific ligand. Therefore, a proper selection of the dimension of separation, as well as the search for new parameters to distinguish hitherto inseparable molecular species, are matters of much interest to those working on biological macromolecules.

Osterman's manual of research methodology gives exhaustive descriptions of electrophoresis, isoelectric focusing and ultracentrifugation (Vol. 1), and immunoelectrophoresis and the application of radioisotopes (Vol. 2). The two books should be extremely useful even to novice researchers; thorough and systematic description of the techniques and plentiful illustrations should enable both graduate students and more experienced researchers to perform high-resolution experiments with the most suitable dimensions of separation. The coverage of the techniques and the references are comprehensive and current to the end of 1982 .

The books should also help in the choice of the most suitable combination of the various separation techniques to obtain results which could not be obtained with one alone, for example two-dimensional display by O'Farrell's method. The quality of the results emerging from such experiments ultimately depends on the individual scientist's skill and training, but these two volumes provide an excellent starting point.

I have one plea. Various types of chromatography are equally important to macromolecular fractionation. In a future edition Dr Osterman might consider including them to make his manual even more complete and valuable.

Akiyoshi Wada is Professor of Biophysics at the University of Tokyo. 